\title{
Models of Preconception Care Implementation in Selected Countries
}

\author{
Shahul H. Ebrahim - Sue Seen-Tsing Lo • \\ Jiatong Zhuo $\cdot$ Jung-Yeol Han $\cdot$ Pierre Delvoye $\cdot$ Li Zhu
}

Published online: 9 June 2006

(C) Springer Science+Business Media, Inc. 2006

\begin{abstract}
Globally, maternal and child health faces diverse challenges depending on the status of the development of the
\end{abstract}

These country examples were presented at the 1st National Summit on Preconception Care, June 21, 2005, Atlanta, GA.

Contributions: SE conceived the idea and country authors provided country specific information for respective sections. Country authors were responsible for assuring the accuracy of information relevant to the respective country examples. SE and JZ contributed to the bulk of the introduction, summary and conclusions. All authors read and approved the final draft.

Disclaimer: The views expressed in this paper are that of the authors and do not necessarily reflect the views of the US Department of Health and Human Services, or the institutions where the authors are employed.

S. H. Ebrahim $(\bowtie)$

Centers for Diseases Control and Prevention,

Atlanta, GA 30333

e-mail: sbe2@cdc.gov

\section{S. S.-T. Lo}

The Family Planning Association of Hong Kong,

Hong Kong

J. Zhuo

Guangxi Centers for Disease Control,

Nanning, PRC

J.-Y. Han

Sungkyunkwan University,

Seoul, Korea

P. Delvoye

Free University of Brussels and ONE (Office for Birth and Childhood),

Belgium

L. Zhu

Peking University Health Science Center,

Beijing, PRC country. Some countries have introduced or explored preconception care for various reasons. Falling birth rates and increasing knowledge about risk factors for adverse pregnancy outcomes led to the introduction of preconception care in Hong Kong in 1998, and South Korea in 2004. In Hong Kong, comprehensive preconception care including laboratory tests are provided to over 4000 women each year at a cost of $\$ 75$ per person. In Korea, about $60 \%$ of the women served have known medical risk history, and the challenge is to expand the program capacity to all women who plan pregnancy, and conducting social marketing. Belgium has established an ad hoc-committee to develop a comprehensive social marketing and professional training strategy for pilot testing preconception care models in the French speaking part of Belgium, an area that represents 5 million people and 50,000 births per year using prenatal care and pediatric clinics, gynecological departments, and the genetic centers. In China, Guangxi province piloted preconceptional HIV testing and counseling among couples who sought the then mandatory premarital medical examination as a component of the three-pronged approach to reduce mother to child transmission of HIV. HIV testing rates among couples increased from $38 \%$ to $62 \%$ over one year period. In October 2003, China changed the legal requirement of premarital medical examination from mandatory to "voluntary." This change was interpreted by most women that the premarital health examination was "unnecessary" and overall premarital health examination rates dropped. Social marketing efforts piloted in 2004 indicated that $95 \%$ of women were willing to pay up to RMB 100 (US\$12) for preconception health care services. These case studies illustrate programmatic feasibility of preconception care services to address maternal and child health and other public health challenges in developed and emerging economies. 


\section{Introduction}

The Task Force on child health and maternal health for the United Nations' Millennium Development Goals reiterated the challenges of achieving the goals in its report "Who's got the power? Transforming health systems for women and children [1]." While the technology and interventions exist to prevent or treat the vast majority of adverse health conditions that affect children and women of reproductive age, many continue to die and even more suffer from ill health $[2,3]$. The central challenge to achieving these goals is to provide known interventions to the full population and at the right time when most known adverse outcomes can be prevented.

Despite 15 years of the global 'Safe Motherhood Initiative,' maternal mortality remains unchanged in that about 530,000 deaths occurred in 2002 [4]. Maternal and child health is connected to social, economic, and environmental conditions. In resource-poor countries, aside from preexisting risks for poor maternal and child health outcomes, global changes have introduced new risks to the health of women and pregnancy, including alcohol and tobacco use, over nutrition, and micronutrient malnutrition $[5,6]$. Because not all the deficits in achieving adequate prenatal care are likely to be solved in the near future, and global factors that lead to increases in risk cannot be reversed, new strategies and policies to improve pregnancy outcome remain a high priority.

One emerging policy is the role of preconception care as a complement to prenatal care [2]. This report highlights examples of national programs and projects indicating that such approaches are feasible and acceptable to the population. These case studies on preconception care that were presented at the First National Summit on Preconception Care, Atlanta, USA, in 2005, range from a mature program in Hong Kong, to emerging programs in Korea and Belgium, and select program enhancement and recovery efforts in China.

\section{Hong Kong: A done deal}

The Family Planning Association of Hong Kong (FPAHK) was founded in 1950 with an objective to reduce family size. The average number of children per woman declined from 4.5 in 1965 to 0.93 by 2004 [7]. Having achieved a low birth rate, a number of sexual and reproductive health programs have been launched with the objective of improving reproductive outcomes. The pre-pregnancy preparation service was launched in 1998, in response to public demand for preconception care. The service integrates-medical, counseling, and education services for couples planning conception to achieve the following outcomes:

1. Prevent and treat infections to safeguard the health of both partners and the fetus. Sexually transmissible infections are screened and treated. Women are tested for rubella antibody and those susceptible are offered vaccination. Partners of hepatitis B virus carriers are offered vaccination. HIV testing is offered as an opt-in test.

2. Screening for thalassemia. Thalassemia is an important hereditary disease in Hong Kong, because approximately $8 \%$ of persons in the local population are heterozygous carriers of mutations for thalassemia [8]. Couples with family history of hereditary diseases are referred for genetic counseling.

3. All women are advised to consume folic acid.

4. Help couples understand their health and adjust their lifestyle to optimize pregnancy outcome.

5. Identify women who might have difficulty conceiving and to advise them to seek sub-fertility investigations early when indicated. For men, semen analysis is included in the package.

6. Help couples prepare for parenthood.

7. Help women with pre-existing medical illness understand the best timing for pregnancy, the effect of their illness on their pregnancy, the effect of pregnancy on their illness, the effect on her offspring, the need to adjust medication, and to avoid drugs with known or uncertain teratogenic effects.

8. Help women with gynecological diseases understand the impact of their diseases on conception and pregnancy. The role of assisted reproductive technology is discussed if appropriate.

9. Counsel couples with coital failure to help them consummate marriage.

At the first visit, the woman fills in a health assessment checklist that include preexisting medical conditions and medication being taken, adverse gynecological history and history of abnormal pregnancy outcomes, and risk behaviors. Couples are then given an educational video compact disc to take home. This disc provides detailed information about the objectives of the service; the physical and genital checkup procedures; pregnancy preparation, e.g. optimal body mass index, balanced diet, regular exercise, quit smoking and avoiding alcohol; fertility regulation including contraception, conception and sub-fertility; and information about the laboratory tests including HIV testing. A nurse then draws blood for testing. At the second visit, a nurse will explain all the normal reports first and then a physician performs the history, physical examination, and counseling to explain any abnormal results. The basic counseling and assessment is provided by non-specialists, although women with complicated problems are seen by specialists in obstetrics and gynecology or an internist. Regular case conferencing 
is conducted to enhance knowledge of the non-specialists and improve their counseling skills.

This is an economically self-sustainable program. The service fee is $\$ 600$ Hong Kong Dollars (USD \$75) per couple which includes laboratory tests and one medical consultation. This low service fee is achieved through cost sharing with other sexual and reproductive health programs offered by the FPAHK. Each year, around 4000-5000 individuals use the service. The continuous flow of clients for the preconception service reflects public support and trust for the service.

\section{South Korea: An evolving initiative}

As may be seen in many emerging economies, South Korea is experiencing decreasing birth rates (from 16.3 per 1000 live births in 1994 to 9.8 per 1000 live births in 2004) and decreasing infant mortality rates (from 9.9/1 ive births in 1993 to 4.6/live births in 2004) [9]. Due to a high rate of unintended pregnancy, an increase in the rate of preterm delivery, a high rate of pregnancy termination following accidental exposure to potential teratogens during pregnancy, and low intake of folic acid, the Society of Maternal and Fetal Medicine made a decision to promote and enhance preconception care in South Korea. The goals are to enhance primary prevention of birth defects and preterm birth, increase in intended pregnancy, decrease in unnecessary pregnancy termination, and recovery of a higher birth rate in Korea to maintain population replacement levels.

The program has two components: increase awareness and service delivery. Education includes lectures for obstetricians and gynecologists as well as for child-bearing age women attending maternity services. Service delivery is clinic-based preconceptional counseling, medical protocol examination by a physician, and follow-up of women who want to have a baby. The Preconception Counseling Clinic, based at the Korean Motherisk Program at Samsung Cheil Hospital, an affiliated University Hospital, includes teratogen information service for pregnant and breast feeding women, as well as preconception counseling.

The preconception care medical protocol is composed of a physical examination, laboratory tests, and a questionnaire. Of the women served, $40 \%$ reported no history of adverse reproductive outcomes, about $30 \%$ had history of spontaneous abortion, $13 \%$ had maternal disease, $15 \%$ had a baby with birth defect, and $2 \%$ had family history of genetic disease. However, careful review of medical and behavioral history indicated that $92 \%$ of preconception care attendees have at least one risk factor for adverse outcomes. Patients with specific problems such as a previous child with a birth defect are transferred to the Department of Maternal and Fetal Medicine. Also, pre-implantation genetic diagnosis is performed in the Department of Infertility, a service that predates the introduction of preconception care. This service resulted in a substantial reduction in pregnancy loss from $91 \%$ in 1995 to $19 \%$ to 203 among 260 couples with balanced chromosomal translocation.

The Korea Motherisk Program is supported by the Samsung Chiel Hospital and is staffed by three University faculty members and rotating fellows in clinical medicine. A researcher has been paid by government research funds to evaluate the program. Although no consultation fee is charged to the patients, laboratory expenses are paid for by the patients. Depending on family history and other factors and various rationale used for laboratory examinations, the maximum cost of such laboratory examinations can be as high as 150 USD per person. Since the establishment of preconception services in 2004, 132 women have been served. The current challenge is to market it to all women who plan pregnancy, and to assure that the health care system has the capacity to match the demand and expansion of the service to other parts of the country.

\section{Belgium: Baby steps}

The O.N.E ("Office de la Naissance et de l'Enfance" = "Organization for Birth and Childhood"), is a Belgian governmental organization charged with the protection and promotion of health of women and children. Given the low fertility rates and high rates of birth defects ( 2 to $3 \%$ of all live births) in Belgium [10], O.N.E launched a plan to assess the feasibility of implementation of preconception care in the French speaking part of Belgium, the region with a population of 5 million people and about 50,000 births per year. This activity will be implemented through prenatal care, pediatric and gynecology clinics, as well as general practitioners, in partnership with genetic and counselling centers.

An ad-hoc committee was formed to propose strategies, activities, financing options, and evaluation methods, starting with the development of evidence-based guidelines based on Belgian epidemiological data. The committee proposed that the guidelines would be in accordance with the recommendations of the Advisory Committee of Bioethics of Belgium concerning genetic tests. The committee proposed also to organize a campaign targeting both the general public and health care providers to help market preconception care in primary care settings.

The proposed guidelines include various serologic tests, screening of endocrine or genetic diseases, and preventive services including promotion of folic acid supplementation. The O.N.E. developed a sustainable campaign plan to inform and sensitize the population about the benefits of preconception care, directing their efforts to individuals aged 15 to 45 years, health professionals including general practitioners, gynaecologists, paediatricians, midwives and all medical 
social workers, members of the family planning centres, and school health promotion centers.

The first step in the social marketing campaign was the creation of the tools including folders, posters, and letters to professionals. These materials were reviewed by the adhoc committee and field tested among a selected group of social workers and the public. The second step will be an information campaign among men and women of reproductive age using posters, folders, and radio and television advertisements. Medical and social workers will also receive folders and posters for distribution to the target population and a letter to inform them about the purpose and methodology of the campaign. Medical practitioners will then receive the guidelines to help them with information to be shared with patients and the laboratory examinations to be conducted. The final step will be the evaluation process to assess behavioral changes both within the community and at provider level. The indicators will include advice received from providers by clients in participating facilities, referral of relevant clients to preconception visits by providers, uptake of preconception visits by clients, and compliance with suggested interventions by clients. The early phase of implementation of preconception care in primary care settings in Belgium was a challenge, as it highlighted the need for a strong partnership of all institutions involved in maternal and child health care.

China: Preconception care as an HIV/AIDS intervention model

A three-pronged approach to reducing mother-to-child transmission of HIV has long been advocated by global initiatives to reduce the burden of HIV/AIDS, but to our knowledge, never been implemented anywhere [11]. This approach includes reduction of HIV infection among young couples through primary prevention and voluntary HIV counseling and testing (VCT), voluntary avoidance of pregnancy by HIV infected couples, and finally antiretroviral treatment of pregnant women who are infected and subsequent management of outcomes in children. The unique premarital medical examination system prevalent in China [12] and late onset of HIV transmission in China provided an opportunity to pilot implementation of the three-pronged strategy in Guangxi province.

Pilot experience in Babu county in Guangxi Province is outlined here. Babu County (population 0.93 million) of Hezhou City, Guangxi Province (population 48.9 million) experienced the fastest increase in reported HIV/AIDS cases in Guangxi Province between 1998 (18) and 2002 (629) since the first case was detected in 1997. Initially, the epidemic predominantly affected male drug users. By 2002, the male to female ratio narrowed to $4: 1$. The epidemic concentrated among the younger age group (90\%, age $20-49$ years) of the population and predominantly rural farmers $(70 \%)$. Given the demographics of the affected population and because the condom usage rate was low in this population (STD clients $5 \%$, drug users $5 \%$, sex workers $40 \%$ ), the potential for mother-to-child transmission was evident.

To reduce the impact of HIV on women, children and families, the county health department in 2001 embarked on a three-pronged approach embedded in the mandatory premarital medical examination system. A feasibility survey in the region had indicated that the majority of the population and public health community were supportive of the concept [11]. The activities included family and community awareness, preconceptional voluntary HIV counseling and tests for couples (PVCT), and VCT and antiretroviral therapy to HIV infected pregnant women. To facilitate PVCT and the antenatal VCT and antiretroviral treatment component, 40 antenatal and maternal/child care clinicians were trained. To facilitate the broad based interventions, 942 medical and public health professionals and barefoot doctors were trained on VCT and other prevention approaches. To facilitate the community engagement process, training seminars using a combination of entertainment and educational films and media slides were conducted in middle schools and high schools for students as well as adults in the catchment's area. An one-time preand post test (8800 respondents) evaluation using structured questionnaire was conducted in selected locations. The students were required to take educational materials $(100,000$ copies) to their homes to educate parents and neighbors as part of their homework requirements. Information on HIV and VCT opportunities were displayed on the community information blackboard, which is the usual tool for disseminating information on public health, legislative, and social policies to villagers and updated frequently by community volunteers.

In the first quarter after launching the program (October to December 2002) an average of 1099 (73\%) of the 1500 eligible couples each month sought PVCT consultation, and 52\% of them (or $38 \%$ of eligible couples) accepted HIV testing. By the end of 2003, the PVCT consultation rate among eligible couples increased to $77 \%$ and HIV testing rate among them reached $80 \%$ (or $62 \%$ of all eligible couples). The main challenge to this approach was the change of legal requirement of premarital medical examination from mandatory to voluntary status in October 2003.

Our pilot efforts indicate that screening for HIV in the preconception period is feasible and acceptable in this rural community. Community sensitization played an important role in its success. A combination of testing strategies implemented at various stages before and during pregnancy would help identify all women at risk for HIV-exposed pregnancies. Given that the community is aware of the concept of premarital medical examination, social marketing techniques using family health enhancement as a core benefit, 
and efforts to regain the conceptual interest of population in preconception care are needed.

China: Regaining the old glory

In October 2003, the longstanding mandatory Chinese requirement for couples planning marriage to obtain a premarital health check, a routine physical examination, laboratory tests, and reproductive health education [12], was removed, and this visit became voluntary. Reported rates of premarital examinations plummeted, and health officials voiced concern that women were not receiving adequate risk assessment and pre-pregnancy education, particularly regarding the benefit of folic acid in preventing neural tube defects.

To assess the feasibility of increasing the rate of preconception care uptake, we conducted a pilot social marketing effort in a northern county (Mancheng, in Hebei Province) during February and March, 2004. We used a three-stage process: 1) ascertain the nature of the problem regarding delivery of preconception care through the use of a series of questionnaires and interviews, 2) develop a test plan to promote preconception care, and 3) evaluate and refine the plan. Overall, $91 \%$ of 10,000 questionnaires were completed, and 140 subjects were interviewed, including women of reproductive age, maternal and child health workers, and local government officials.

The major obstacles to preconception care included the following: a) lack of systematic organization of these services within the health care system; b) poor coordination between governmental organizations involved in marriage, family planning and health care; c) unclear media messages; and d) the perception of the term "voluntary" by women to mean "unnecessary." The implementation plan included the use of social marketing to solicit government support, coordinate with various government organizations, train maternal child health $(\mathrm{MCH})$ workers at all levels, and to market the concept of preconception health care to women of childbearing age. To solicit government support, we established a project coordination committee, including high-level officials from the county project office, the county women's office, the bureaus of health, family planning, and civil administration, and the women's federation. In addition, a series of face-to-face interviews were conducted with government officials, workshops were held in the county, and an official document was issued by the county government. Recommendations were developed for improving coordination among government departments and organizations, including development of an education program that can be presented by the $\mathrm{MCH}$ institute, the marriage registration office, and the family planning office, whenever women have contact with the system. Such recommendations were shared with all stakeholders. Following the workshop, 95\% of women reported that they would be willing to pay up to
RMB 100 (US\$12) for preconception health care services, and half indicated that more and better health messages were needed.

Improving coordination among government agencies, developing training materials targeted to different groups, and promulgating clear, consistent messages concerning the importance of preconception care that are delivered in all settings where women may have contact with the system before pregnancy can create demand for preconception services.

\section{Conclusions}

As illustrated by the six examples highlighted above, it is evident that preconception care is not just a conceptual debate but a primary approach used to address various health issues and emerging national challenges. As noted so cogently by Atrash et al. [2] a healthy baby and a healthy mother are valued hopes and dreams of families and cultural heritages across the world. Further, in the countries such as South Korea and Hong Kong, and others in similar demographic transition, this "valued hope" also represents national strategy perspective.

Emphasis on preconception care should not be limited to developed and emerging economies. Globalization of health risk factors is a phenomena that affects health of all nations. Although adequate prenatal, obstetric, and primary care services can reduce the infant and maternal mortality in high-mortality-developing countries, such countries are not free from emerging risks to maternal and child outcomes. Data from 36 developing countries showed that overweight now exceeds underweight among women [5]. Tobacco and alcohol use rates among women in many developing countries have increased [13]. However, as more women than before have access to education and information, are employed, have personal income and decision making power, and delay pregnancy, there are many opportunities to inform them about the need for preconception care and a healthy reproductive life.

Indeed, even a perfectly organized preconception care system is not likely to address other deficits in the reproductive health care system or in an inadequate prenatal care system in developing countries; neither will it lead to rapid positive changes in related indicators. Similar to the arguments in 1990s for and against HIV voluntary counseling services amidst the lack of availability of antiretroviral treatment, or against the attempts to market cell phones where land phones services are inadequate [13], advocating preconception care as a component of maternal child care services will remain controversial. As such, establishment of preconception care services need not wait for prenatal care system to be adequate or perfect. In fact, introduction to preconception care can complement the efforts to improve prenatal 
care uptake. Consistent with the conceptual framework of the 2005 Bangkok charter on health promotion [14], preconception care should be seen as a program for the future, a development agenda that is aimed at overall health of the family. Making preconception care available can have transgenerational impact on some women; those who are aware of such opportunities and can access such services. Our responsibility is to provide that opportunity.

\section{References}

1. UN Millennium Project 2005. Who's got the power? Transforming Health Systems for Women and Children. Summary version of the report of the Task Force on Child Health and Maternal Health, New York, USA.

2. Atrash H, Adams M, Cordero J, Howse J. Time to act on missed opportunities to improve perinatal outcomes. Mat Child Health Journal; 2006: (this edition).

3. Jones G, Steketee R, Black R, Bhutta ZA, Morris S and the Bellagio Study Group on Child Survival. How many child deaths can we prevent this year? The Lancet 2003;362:65-71.

4. WHO, UNICEF, and UNFPA 2004. Maternal mortality in 2000, estimates developed by UNICEF, WHO, and UNFPA. World Health Organization, Geneva Switzerland.
5. Mendez M, Monteiro C, Popkin B. Overweight exceeds underweight among women in most developing countries. Am J Clin Nutr 2005;81:714-21.

6. World Health Organization. The World Health Report 2002. Reducing risks, promoting healthy life. WHO, Geneva, 2002.

7. http://www.info.gov.hk/censtatd/eng/hkstat/index.html. Accessed on April 22, 2005.

8. Lau YL, Chan LC, Chan YY, Ha SY, Yeung CY, Waye JS, Chui DH. Prevalence and genotypes of alpha- and beta-thalassemia carriers in Hong Kong - implications for population screening. N Engl J Med 1997;336:1298-301

9. http://www.nso.go.kr/newnso/s_data/j_potal_view.html?category_ id =108), accessed September 1, 2005.

10. http://www.one.be/home.htm, accessed August 30, 2005.

11. Abdullah AS, Guan FQ, Zhuo J, Zhang SX, Geng W, Ebrahim SH. Need, readiness and opportunities for family HIV/AIDS intervention in China. Int J STD AIDS 2003; 14:642-3.

12. Hesketh T. Getting married in China; pass the medical first. BMJ 2003;326:277-9.

13. Canadian International Development Agency. Grammeen Telcoms Village Phone Programme: A multimedia case study. Ontario, Canada, 2000.

14. BMJ. Who conference calls for health promotion to be at center of development. BMJ 2005;331(442):422. 